\title{
Chilla, T.; Kühne, O.; Neufeld, M. (2016): Regionalentwicklung
}

\author{
Stuttgart: Verlag Eugen Ulmer. 289 S., 16 Tab., 93 Abb.
}

\author{
Ingo Mose $^{1} \mathbb{D}$
}

Online publiziert: 19. Juli 2017

(c) Springer-Verlag GmbH Deutschland 2017

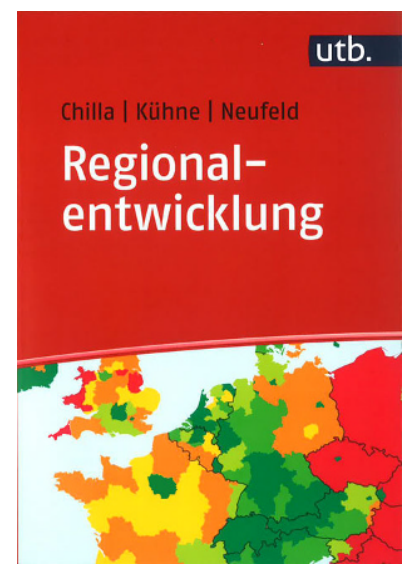

Kein Bücherregal Studierender, in dem nicht wenigstens einige der roten Bände der UTB-Reihe stehen würden. Generationen von Studierenden, aber auch Lehrenden sowie Praktikern verschiedenster Provenienz haben Gebrauch von den gleichermaßen bekannten und anerkannten Lehrbüchern gemacht, deren Qualität nach wie vor allseits geschätzt wird. Und jährlich wird die Reihe um neue Themen erweitert. Im vergangenen Jahr erschien im Verlag Eugen Ulmer ein Band zum Thema „Regionalentwicklung“, mit dem die Serie raumwissenschaftlicher Lehrbücher in der UTB-Reihe um einen besonders facettenreichen Gegenstand erweitert worden ist. Erstmals, so bewirbt der Verlag den Band, sei damit ein Lehrbuch erschienen, das eine

Prof. Dr. Ingo Mose

ingo.mose@uni-oldenburg.de

1 Arbeitsgruppe Angewandte Geographie und Umweltplanung, Zentrum für nachhaltige Raumentwicklung in Oldenburg - ZENARiO, Carl von Ossietzky Universität Oldenburg, Ammerländer Heerstraße 114-118, 26239 Oldenburg, Deutschland umfassende und systematische Darstellung zur Regionalentwicklung biete.

Die Autoren, die für den Band verantwortlich zeichnen, Tobias Chilla, Markus Neufeld (beide Friedrich-Alexander-Universität Erlangen-Nürnberg) und Olaf Kühne (Eberhard Karls Universität Tübingen), haben in ihrem Vorwort die Erstellung eines Lehrbuchs zur Regionalentwicklung als „,wirkliche Herausforderung“ (S. 9) charakterisiert. Das mag für Lehrbücher generell stimmen, für dieses Thema trifft es aber zweifellos in besonderer Weise zu. Für wenige Gegenstände der Raumwissenschaften gilt, dass sie gleichermaßen konzeptionelle wie normative Zugänge implizieren, eine Vielzahl von Akteuren betreffen, ein Instrumentarium auf unterschiedlichsten räumlichen Maßstabsebenen thematisieren und verschiedenste Handlungsfelder ansprechen wie die Regionalentwicklung. Damit handelt es sich nicht zuletzt um einen interdisziplinären Gegenstand, der seine Inhalte aus verschiedenen Disziplinen auch jenseits der Raumwissenschaften gewinnt und mit dem Ziel einer holistischen Betrachtung sinnvoll integriert.

Nicht zuletzt ihren langjährigen Erfahrungen als Forscher und, vor allem, als Lehrende können es die Verfasser danken, dass sie ein Lehrbuch geschrieben haben, das diese Bezeichnung zweifellos verdient. Seine positiven Qualitäten sind schnell benannt: Eine klare, systematische Gliederung strukturiert den Gegenstand nachvollziehbar und überzeugend und dient der leichten Orientierung bei der Lektüre. Die textlichen Darstellungen sind durchweg sehr gut lesbar, verständlich und ansprechend. Dabei werden, so mein Eindruck, die Ansprüche unterschiedlicher Studienniveaus, Anfänger und Fortgeschrittene, gleichermaßen gut bedient. Zahlreiche Abbildungen und Tabellen, viele davon in Farbe, illustrieren und unterstützen den Text in vorteilhafter Weise. Als besonders gelungen erachte ich die punktuelle Vertiefung verschiedener Aspekte anhand ausge- 
wählter Beispiele, die sich wie an einem roten Faden durch das gesamte Buch ziehen. Viele der gewählten Beispiele verweisen auf ebenso prominente wie anschauliche ,Fälle', zeichnen sich aber auch durch ein hohes $\mathrm{Ma} ß$ an Aktualität aus. Regionalentwicklung wird dabei primär aus der Perspektive Deutschlands thematisiert, ohne zugleich die nötige Einbettung in den europäischen Kontext zu übersehen. Ein recht umfangreiches Literaturverzeichnis (mit deutschebenso wie englischsprachigen Titeln) bietet schließlich die Grundlage zur eigenständigen Vertiefung.

Mit anderen Lehrbüchern vergleichbarer Art teilt der vorliegende Band das Problem, dass viele der verwendeten Daten, seien es die regionalen Kaufkraftstandards, die Grunderwerbssteuersätze der Bundesländer, die Übernachtungszahlen touristischer Destinationen oder die Fördergebietskulisse der europäischen Strukturfonds, der fortlaufenden Veränderung unterliegen und in absehbarer Zeit veraltet sein werden. Diese Schwäche wiegt gleichwohl nur bedingt schwer, als viele Inhalte, wie etwa die konzeptionellen Grundlagen oder das Instrumentarium der Regionalentwicklung, sich durch einen vergleichsweise ,zeitlosen Charakter' auszeichnen. Bei der Breite der angesprochenen thematischen Aspekte, die dem ambitionierten Anspruch auf eine umfassende Darstellung des Gegenstands geschuldet ist, konnten es die Verfasser, gewissermaßen vorhersehbar, nicht vermeiden, einige Inhalte stark zu komprimieren - und damit gelegentlich etwas oberflächlich zu bleiben. Gleichwohl bringt es das Buch auch so auf fast 300 Seiten. Viele der zahlreichen beispielhaften Illustrationen, wenn auch selber teils sehr komprimiert, helfen diese Schwäche auszugleichen. Auffällig häufig ist der Verweis der Verfasser - sicherlich bedingt durch ihre Biographien auf (eigene) Erfahrungen aus Bayern und Süddeutschland, während der Norden des Landes in Summe leider etwas ,unterbelichtet' bleibt. Wenn auch nur eine Marginalie, so doch der Erwähnung wert, ist die uneinheitliche Ansprache ländlicher Räume im Singular und Plural; sie sollte unbedingt einheitlich im Plural erfolgen, um damit der Vielfalt regionaler Entwicklungsprozesse und -muster Rechnung zu tragen! Eher überrascht als kritisch anzumerken ist die Beobachtung, dass dem Literaturverzeichnis eine Reihe einschlägiger Publikationen zu Themen der Regionalentwicklung, beispielsweise zur ländlichen Regionalentwicklung und zum Zusammenhang von Regionalentwicklung und Naturschutz, fehlt. Nichts anderes belegt dieser Umstand, als dass letztlich kein Buch, so anspruchsvoll es auch konzipiert und so sorgfältig es auch recherchiert sein mag, wirklich ,umfassend" sein kann. Inwieweit auch grundsätzlich eine andere (womöglich speziellere) Konzeptualisierung des Gegenstandes denkbar wäre, auf deren Grundlage entsprechend auch ein anderes Lehrbuch entstünde, mag an dieser Stelle dahingestellt bleiben.

Der wenigen erwähnten Schwächen eingedenk, sei den Autoren abschließend zu ihrer Publikation aufrichtig gratuliert: Ein Lehrbuch zur Regionalentwicklung in einer breiten Perspektive, wie sie ihrem UTB-Band zugrunde liegt, hat bisher in der Tat gefehlt. Als Lehrbuch ebenso wie als Nachschlagewerk sei es Studierenden der raumwissenschaftlichen Disziplinen ebenso wie Praktikern aus dem Berufsalltag der Regionalentwicklung zur Lektüre gerne empfohlen. 